\title{
Variabilité pluviométrique, changement climatique et régionalisation en région de mousson africaine
}

\author{
Bernard Fontaine $^{(1)}$, Pascal Roucou ${ }^{(1)}$, Moctar Camara $^{(2)}$, \\ Nicolas Vigaud ${ }^{(1)}$, Abdourahamane Konaré ${ }^{(3)}$, Seidou lbrah Sanda ${ }^{(4)}$, \\ Arona Diedhiou $^{(5)}$ et Serge Janicot ${ }^{(6)}$ \\ (1) CRC, UMR6282 Biogéosciences, CNRS/Université de Bourgogne, \\ 21000 Dijon, France \\ (2) Laboratoire d'Océanographie, des sciences de l'environnement et du climat \\ (LOSEC), Université de Ziguinchor, BP 523, Ziguinchor, Sénégal \\ (3) Laboratoire de physique de l'atmosphère et de mécaniques des fluides \\ (LAPA-MF), 22 BP 582 Abidjan 22. Université de Cocody, Abidjan, \\ Côte d'Ivoire \\ (4) Département de Physique, Université Abdou Moumouni de Niamey, \\ BP 10662, Niamey, Niger \\ (5) LTHE, UMR5564, IRD/CNRS, Université de Grenoble, Grenoble, France \\ (6) LOCEAN, UMR7159, IRD/CNRS/P6, Paris, France
}

\section{Résumé}

Cette synthèse rappelle quelques résultats à l'issue de l'expérience internationale AMMA (2003-2010) en matière de variabilité de la mousson africaine (des échelles intra-saisonnières à pluri-décennales) et de prospective climatique. Ceux-ci ont confirmé le poids des températures de surface marine et des téléconnexions pour les fluctuations interannuelles et décennales tout en réévaluant l'importance de la variabilité interne de l'atmosphère. Ils ont aussi précisé les modes dominants de variabilité intrasaisonnière et les interactions avec les états de surface. Plusieurs hypothèses impliquant les effets mémoire liés à l'eau du sol et à la végétation, particulièrement au printemps et en automne boréal, ont par ailleurs été formulées. En termes de prospective, l'analyse des sorties de modèles suggère plutôt des excédents de précipitations sur le Sahel central et oriental et un déficit relatif plus à l'ouest vers 2050 . La phase 2 d'AMMA (2010-2020) s'attachera davantage aux aspects qui ont un fort impact sociétal en collaboration directe avec les services météorologiques : prévisibilité, scores de prévision, indicateurs opérationnels, évaluation de la part du forçage anthropique dans l'évolution actuelle et future. a gamme de variabilité climatique, allant de l'échelle intra-saisonnière (supérieure à 10 jours) aux échelles décennale et pluri-décennale, impacte les écosystèmes et les activités humaines, particulièrement sur le continent africain où la dépendance des sociétés aux aléas naturels, en premier lieu pluviométriques, est la plus forte. La diminution tendancielle observée sur les cumuls pluviométriques de la mousson d'été sur le Sahel entre les années 1950 et 1980 et plus encore la succession quasi ininterrompue d'années anormalement sèches depuis la fin des années 1960 ont marqué les mémoires (figure 2a), interrogé les scientifiques et démontré la nécessité de mieux connâ̂tre et documenter la variabilité de la mousson africaine. Cet épisode anormalement long a enregistré un déficit cumulé moyen allant de 15 à $50 \%$, une diminution du nombre des événements pluvieux les plus intenses, malgré une reprise partielle depuis le milieu des années 1990 sur le centre et l'est du Sahel, l'ouest demeurant plus sec (Lebel et Ali, 2009).

L'une des priorités d'AMMA a donc été de préciser les caractéristiques multiéchelles de la variabilité de la convection et des précipitations afin de pouvoir améliorer le diagnostic sur observations et l'analyse climatique au travers d'expériences forcées et couplées sur les modèles de circulation générale. Les campagnes de terrain, menées entre 2005 et 2007 à la fois dans l'atmosphère, sur le continent et dans l'Atlantique tropical et équatorial, ont par exemple permis d'obtenir des flux de chaleurs plus fiables et de mieux fermer les bilans d'énergie. On a pu ainsi identifier selon les échelles les principaux processus physiques et dynamiques impliqués en recourant aux modèles non hydrostatiques à aire limitée. À l'issue du déroulement de la phase 1 d'AMMA, il est utile de présenter l'état des connaissances dans ce domaine en rappelant simplement quelques-uns des résultats obtenus et les limites associées selon les différents facteurs et les échelles de variabilité impliqués, ainsi que les hypothèses formulées pour le futur.

\section{Variabilité : diagnostics et facteurs impliqués}

La phase 1 d'AMMA a permis de confirmer que la variabilité de la mousson africaine résulte d'abord de la variabilité interne propre à l'atmosphère aux pas de temps courts et synoptiques, et aux échelles supérieures, des interactions 


\section{$\ldots / . .$.}

\section{Abstract}

\section{Rainfall variability, climate change and regionalization in the African monsoon region}

This summary recalls some results at the end of the AMMA international experiment (2003-2010) in terms of variability of the African monsoon at the intraseasonal to multi-decadal scales and of climate prospective. The results confirmed the weight of surface temperatures and marine teleconnections for interannual and decadal fluctuations and stressed the importance of atmospheric variability. They also described the dominant modes of intraseasonal variability as their interactions with the surface. Several hypotheses involving memory effects related to soil water and vegetation, particularly in boreal spring and autumn have also been made. Prospective analysis from model output suggests rainfall surplus around 2050 over the Eastern-central Sahel and relative deficit to the West. Phase 2 of AMMA (2010-2020) will focus more on aspects that have a high social impact in direct collaboration with meteorological services: predictability, prediction scores, operational indicators, evaluation of the part of anthropogenic forcing in the current and future variations. multiples entre milieu océanique, biosphère continentale et atmosphère dont les dynamiques et mémoires sont très différentes. Au sud du Sahara, ces variations affectent principalement la circulation de mousson à l'échelle continentale et, aux échelles fines, la convection et les précipitations qui contrôlent l'intensité et le déroulé de la saison des pluies d'été boréal centrée sur juillet-septembre. On peut aujourd'hui quantifier la variabilité de la convection au travers des mesures par satellite de rayonnement infrarouge sortant au sommet de l'atmosphère associée aux précipitations : l'ensemble des système convectifs organisés passant sur ces régions représentent $90 \%$ environ du total de saison des pluies d'après Mathon et al. (2002). Ainsi, aux échelles inférieures à 3 jours et synoptiques (3-10 jours), l'analyse de variance des données filtrées à ces échelles montre l'intermittence de ces signaux dans chacune de ces gammes et explique $40 \%$ de toute la variabilité observée aux pas de temps inférieurs à 3 mois, ce qui est plus qu'au sein de la mousson indienne d'après Roehrig (2010). Aux échelles supérieures à 10 jours, les mêmes analyses aboutissent à un taux de variabilité qui n'est plus que de l'ordre de $20 \%$, mais qui a évidemment un impact plus fort en engendrant des anomalies de 1'ordre de 40-80\% par rapport aux valeurs saisonnières moyennes $\pm 4 \mathrm{~mm} /$ jour pour les pluies et de $\pm 15 \mathrm{~W} / \mathrm{m}^{2}$ pour le rayonnement infrarouge sortant (Roehrig, 2010). Dans cette gamme, on peut schématiquement distinguer une variabilité intrasaisonnière entre 10 et 90 jours, et une variabilité interannuelle et décennale.

\section{La variabilité intra- saisonnière (10-90 jours)}

Aux basses latitudes, la variabilité interne de l'atmosphère et les interactions avec la surface continentale, très fortes en Afrique, induisent des perturbations atmosphériques qui vont pouvoir se propager sur de longues distances via les circulations cellulaires et les ondes atmosphériques. Ces propagations principalement zonales prennent parfois un aspect quasi stationnaire lorsque les interactions avec la surface sont dominantes. Elles expliquent pour partie les périodes actives/inactives de la mousson africaine mais aussi l'arrivée de la mousson (« onset» en anglais) qui marque le déport soudain vers le nord, vers la fin du mois de juin, de la zone de convergence intertropicale sur le continent entre la Basse Côte à $6^{\circ} \mathrm{N}$ et le Sahel à $10^{\circ} \mathrm{N}$ (figure 1a).
De même, la dépression thermique saharienne, qui est ressortie des campagnes de terrain et de la modélisation méso-échelle comme étant un élément clé de la circulation de mousson et de la convection au Sahel (Lavaysse et al., 2009), subit les trains d'ondes de Rossby qui se propagent lentement vers l'est au sein des jets polaire et subtropical : ces ondes modulent les transports d'humidité et de température vers le tropique du Cancer ce qui modifie la position et l'intensité de la dépression et affecte la mousson et ses effets pluviométriques (Chauvin et al., 2010).

Enfin, le rôle de la langue d'eaux froides qui se développe en fin de printemps boréal dans le golfe de Guinée a pu être précisé (Caniaux et al., 2011, et ce numéro, p. 17-24). Il a été confirmé que cette langue d'eaux froides est associée à un upwelling répondant à la fois au forçage du vent et à la dynamique océanique, et son phasage avec l'arrivée de la mousson au Sahel a été précisé : celle-ci est plus tardive lorsque des températures océaniques anormalement chaudes dans le golfe de Guinée maintiennent la convergence des vents sur l'océan, impliquant des précipitations plus fortes que la normale sur la Basse Côte (et vice-versa).

Trois principaux modes de variabilité intra-saisonnière ont par ailleurs été isolés au sein de l'activité convective et des précipitations (Janicot et al., 2011) :

1) un dipôle d'anomalies zonales modulant la convection entre l'Afrique et l'Atlantique, centré sur 14-15 jours et répondant à la dynamique de modes atmosphériques équatoriaux vers l'est et d'interactions entre la surface et l'atmosphère (mode " guinéen », figure 1c). Lorsque la convection profonde est minimale sur l'Afrique, la radiation solaire reçue et la température de surface augmentent alors que la pression diminue ; cela favorise l'advection d'humidité depuis l'Atlantique, qui peu à peu autorise la convection profonde et donc la phase inverse ;

2) un mode " sahélien » centré aussi sur 15 jours (figure 1d). Initié en Afrique de l'Est équatoriale cette anomalie convective se déplace sur le Sahel vers $15^{\circ} \mathrm{N}$, puis migre vers l'ouest avant de se dissiper à l'approche de l'Atlantique. Elle est marquée par une anomalie de circulation cyclonique dans les basses couches sur le Sahel générant des vents de sud, ce qui à l'est de $15^{\circ} \mathrm{E}$ augmente l'humidité et donc la convection. Le déplacement vers l'ouest de la structure amène la phase inverse : cellule anticyclonique advectant de l'air sec depuis 


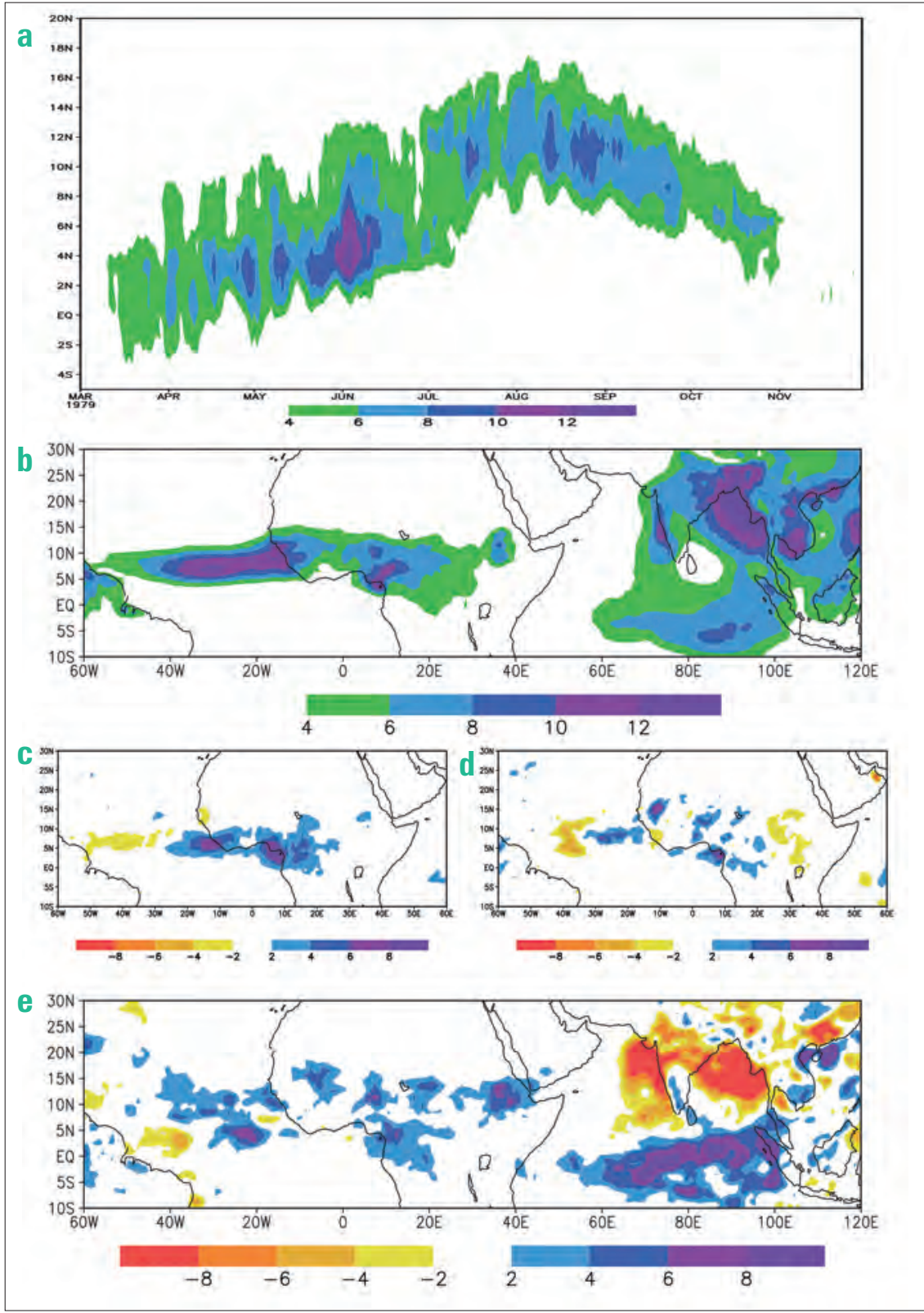

Figure 1 - a) Coupe temps-latitude des précipitations quotidiennes (mm/jour) moyennées entre $5^{\circ} \mathrm{W}$ et $5^{\circ} \mathrm{E}$ sur les années 1997 à 2006 (données du Global Precipitation Climatology Project). b) Moyenne des pluies quotidiennes (mm/jour) sur juin-septembre. c) Mode de variabilité " guinéen » représenté par la différence moyenne de précipitations (mm/jour) entre ses phases fortes et faibles durant l'été. d) Idem à (c) pour le mode " sahélien ". e) Idem à (c) pour le mode " MJO ».

le nord, ce qui limite la convection et module le flux de mousson et le jet d'est africain des couches moyennes mais aussi la dépression saharienne. Ce mode explique en partie l'origine des épisodes secs et humides pendant la saison des pluies sahélienne (Roehrig, 2010). Il résulte principalement, d'une part de la dynamique des ondes équatoriales de Rossby couplées à la convection, et d'autre part des trains d'ondes de Rossby des latitudes moyennes qui descendent vers la Méditerranée et l'Afrique du Nord ; son maintien et sa progression vers l'ouest sont liés aux interactions radiatives avec la surface continentale (Taylor et al., 2011a) ;

3) enfin, des oscillations de l'ordre de 30-90 jours de type Madden-Julian se propageant vers l'ouest (figure 1e). Ces oscillations sont repérées sur l'Afrique entre le nord du Tchad et l'Atlantique, en lien avec des trains d'ondes équatoriales de Rossby couplées à la convection. Elles sont initiées par l'alternance des périodes actives et inactives au sein de la mousson indienne (Matthews, 2004).

La phase 2 d'AMMA aura pour objectifs principaux de mieux comprendre les mécanismes en jeu dans l'initiation, l'intensification et le déplacement de ces structures de convection qui dépassent l'échelle des événements convectifs individuels, d'évaluer leur prévisibilité avec les modèles actuels, d'améliorer les scores de leur prévision et de mettre en place des indicateurs opérationnels en collaboration avec les services météorologiques africains.

\section{Variabilité interannuelle et décennale}

Les analyses spectrales des longues séries de précipitations attestent d'une variabilité entre 2 et 4 ans au Sahel, entre 2 et 8 ans en région guinéenne auxquelles se superposent des oscillations plus lentes (sur 8-16 ans au Sahel, sur 16-18 ans en Guinée) ainsi qu'une évolution multi-décennale (Caminade, 2010). L'originalité d'AMMA a été de mener des observations intensives et des expériences numériques dédiées pour préciser quels étaient les forçages de surface associés à ces échelles. Les résultats ont confirmé que ceux-ci résultaient d'abord de l'influence des températures de surface océanique des différents bassins intertropicaux via des téléconnexions atmosphériques et, au deuxième ordre, des interactions avec la surface continentale (albédo, eau du sol, végétation) qui induisent des effets mémoire et jouent sur la dynamique atmosphérique par des anomalies de subsidence et de vent (jets). Les effets océaniques dominent la réponse des modèles mais il faut dire que ceux-ci prennent mal en compte actuellement l'évolution interannuelle de la végétation et de l'eau du sol en raison de leur incapacité à simuler correctement les précipitations, les flux verticaux et les stocks (Taylor et al., 2011a).

\section{L'océan}

C'est la présence d'eaux anormalement froides dans l'Atlantique nord et anormalement chaudes dans l'Atlantique tropical sud qui a d'abord été associée aux déficits pluviométriques sahéliens, entraînant une montée réduite des pluies vers le nord (Lamb, 1978). Par la suite, on s'est aperçu que cette structure dipolaire atlantique relevait pour partie d'une structure globale interhémisphérique liée au plus fort réchauffement de l'océan tropical et sud enregistré sur le dernier siècle (Folland et al., 1986). AMMA a montré que ce phénomène multi-décennal pourrait être aussi d'origine anthropique, bien que mal simulé dans les modèles couplés (Joly et al., 2007), et que les décennies chaudes sur les parties est-équatoriales de l'Atlantique et du Pacifique ont bien correspondu aux années les plus sèches au Sahel, notamment après les années 1960 (figure 2). D'ailleurs, un golfe de Guinée anormalement chaud maintient les anomalies de convergence du flux d'humidité sur la Basse Côte et limite donc la pénétration continentale du flux de mousson (Caniaux et al., 2011). 


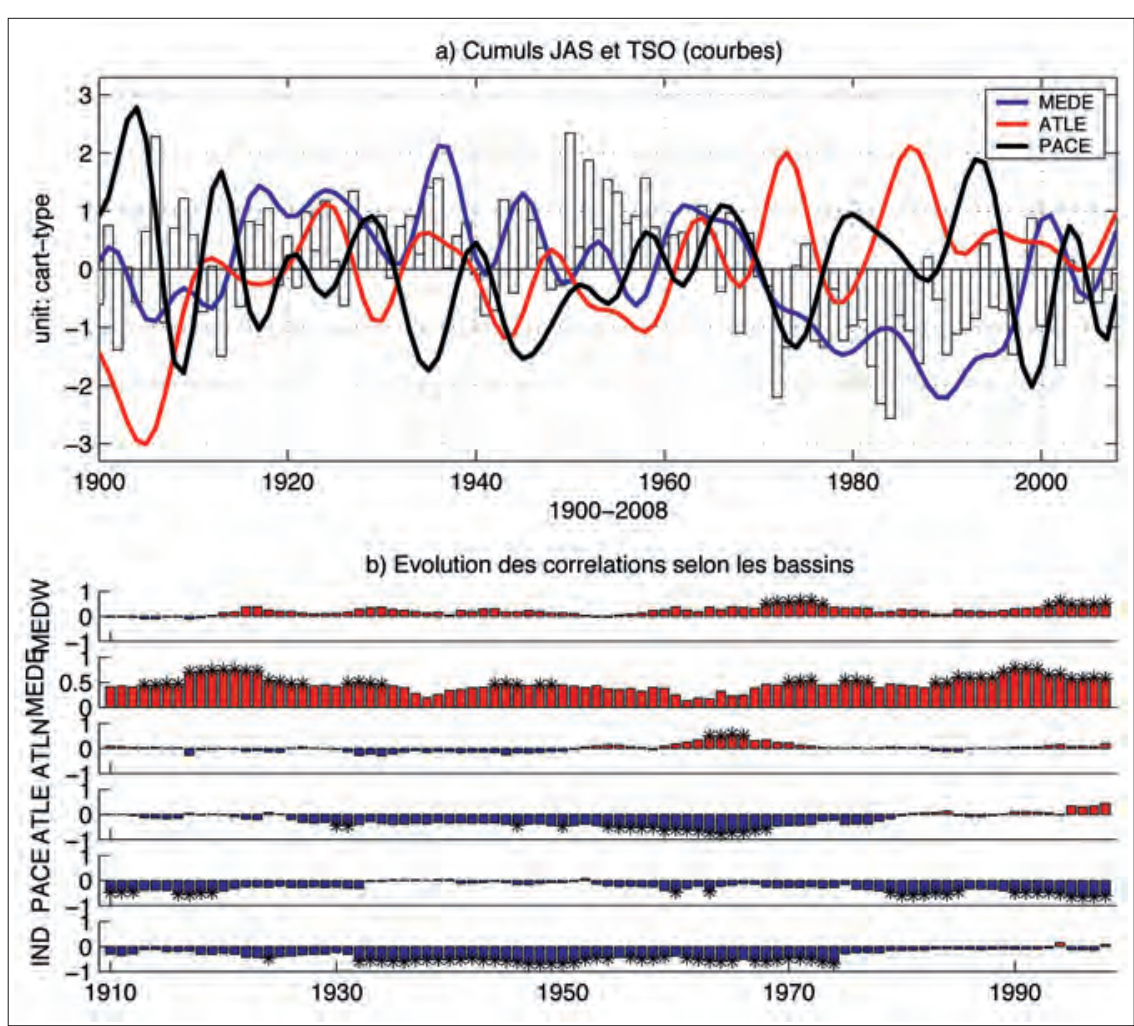

Figure 2 - a) Évolution interannuelle des cumuls sur juillet-septembre au Sahel (barres) comparée à l'évolution quas décennale (supérieure à 8 ans) des températures de surface de la mer pour quelques bassins (courbes) sur la période 1900-2008 : Méditerranée est (MEDE), Atlantique est équatorial (ATLE) et Pacifique central et équatorial (PACE).

b) Évolution des coefficients de corrélation entre précipitations sahéliennes et anomalies des températures par rapport à celles de l'océan global : bassins ouest et est de la Méditerranée (MEDW et MEDE), Atlantique tropical nord et équatorial sud (ATLN et ATLE), Pacifique central équatorial et océan Indien tropical (PACE, IND). Les valeurs sont calculées sur des périodes glissantes de 20 ans (1910 indique donc la période 1900-1919) les étoiles marquent les valeurs significatives au seuil $95 \%$ d'après une procédure de Monte-Carlo (adapté des figures 1 et 2 de Fontaine et al., 2010).

Le programme a aussi précisé les interactions entre bassins et échelles de variabilité temporelle. En effet, l'évolution interannuelle des températures de surface océaniques se compose avec deux oscillations océaniques plus lentes :

1) l'Oscillation multi-décennale de l'Atlantique qui génère des anomalies thermiques très étendues et persistantes dans le bassin nord reflétant en surface les différentes phases de la circulation thermohaline de l'océan global ; Pacifique qui modifie les gradients thermiques à la surface de l'océan et donc les téléconnexions via la circulation atmosphérique de Walker reliant près de l'équateur les bassins océaniques. Ainsi,
2) l'Oscillation quasi décennale du la sécheresse des années 1970 et 1980 a correspondu avec une Oscillation Pacifique positive et une Oscillation multi-décennale de l'Atlantique négative, favorables à une moindre remontée de la zone de convergence sur l'Afrique, alors que les années 1950 et 1960 pluvieuses, comme la récente petite reprise pluviométrique des années 1990-2000, correspondaient à une Oscillation multidécennale de l'Atlantique revenant en phase positive (Mohino et al., 2011).

Enfin, la variabilité interannuelle des anomalies de température du Pacifique équatorial et de la Méditerranée par rapport à l'évolution thermique de l'océan global entretiennent aussi des liens avec la mousson africaine via, respectivement, la circulation de Walker associée aux ondes équatoriales d'altitude et les advections d'humidité depuis la Méditerranée. Les déficits (excédents) enregistrés sur le Sahel central et oriental sont ainsi plutôt synchrones des épisodes El Niño (La Niña) précoces et de la présence d'anomalies froides (chaudes) sur l'est de la Méditerranée. Ces liens statistiques sont non stationnaires comme indiqué sur les diagrammes de la figure $2 b$ : ils sont plus forts avec le Pacifique après 1970, car ils se composent avec l'Oscillation quasi décennale Pacifique (Janicot et al., 2001), mais plus stables avec la Méditerranée, bien que renforcés depuis une trentaine d'années (Fontaine et al., 2011a,b).

C'est donc la superposition de tous ces modes qui explique pour une part importante la variabilité interannuelle et décennale. Le tableau 1 synthétise les mécanismes impliqués dans les téléconnexions avec la Méditerranée, l'Atlantique et le Pacifique (adapté de

Tableau 1 - Principaux mécanismes impliqués dans les téléconnexions entre la Méditerranée (MED), I'Atlantique (ATL) et le Pacifique (PAC), et les précipitations en Afrique de l'Ouest (adapté de Rodriguez et al., 2011).

$\begin{gathered}\text { Forcage } \\ \text { thermigue }\end{gathered}$
MED plus chaude
(surtout bassin est)

\section{ATL plus chaud (surtout partie est équatoriale)}

\begin{tabular}{l|l}
\hline PAC ouest & PAC est \\
équatorial \\
plus froid
\end{tabular}

\section{Méconismes atmosphériques impliqués}

\begin{tabular}{|c|c|c|}
\hline $\begin{array}{l}\text { Plus d'humidité } \\
\text { disponible } \\
\text { avec convergence } \\
\text { des flux sur MED }\end{array}$ & $\begin{array}{l}\text { Plus d'humidité advectée } \\
\text { au sud du Sahara } \\
\text { par la circulation } \\
\text { moyenne }\end{array}$ & $\begin{array}{l}\text { Convergence renforcée } \\
\text { sur le Sahel et flux } \\
\text { de mousson plus intense }\end{array}$ \\
\hline \multirow{2}{*}{$\begin{array}{l}\text { Affaiblissement } \\
\text { des gradients } \\
\text { océan-continent } \\
\text { de température } \\
\text { et de pression }\end{array}$} & \multirow{2}{*}{$\begin{array}{l}\text { Circulation } \\
\text { de mousson } \\
\text { affaiblie } \\
\text { sur le continent }\end{array}$} & $\begin{array}{l}\text { Moins de convergence } \\
\text { sur le Sahel }\end{array}$ \\
\hline & & $\begin{array}{l}\text { Maintien de la convection } \\
\text { anormalement au sud }\end{array}$ \\
\hline $\begin{array}{l}\text { Refroidissement } \\
\text { sur le continent } \\
\text { maritime }\end{array}$ & $\begin{array}{l}\text { Génération } \\
\text { d'ondes } \\
\text { de Rossby }\end{array}$ & $\begin{array}{l}\text { Subsidence } \\
\text { renforcée } \\
\text { sur le Sahel }\end{array}$ \\
\hline
\end{tabular}

\section{Précipitations}

en Afrique de l'Ouest

Positionnement anormalement nord
de la zone de convergence intertropicale
avec excédents sur le Sahel
Déficit aux latitudes sahéliennes
Excédents plus au sud
sur la Basse Côte


Rodriguez-Fonseca et al., 2011). Cependant, Traore et Hourdin (2012) montrent que la variabilité interne de l'atmosphère, indépendante du forçage océanique, peut moduler de façon importante celle de la mousson africaine, même à l'échelle décennale.

\section{La surface continentale}

C'est le célèbre article de Charney (1975) qui, le premier, attira l'attention sur un mécanisme de rétroaction positive pouvant expliquer la persistance interannuelle des anomalies négatives de précipitations en régions subdésertiques aux échelles locale et sousrégionale : une augmentation d'albédo de surface par désertification, en réduisant l'énergie disponible dans la colonne atmosphérique, induit un réchauffement par subsidence (compression adiabatique) qui inhibe la convection et maintient ou déplace la zone de convergence intertropicale vers le sud, l'assèchement de surface consécutif favorisant alors une augmentation additionnelle de l'albédo.

À l'échelle de la circulation de mousson, certaines conditions de printemps boréal qui influencent les gradients de température et d'énergie statique humide en surface, et d'autres en automne qui jouent sur l'eau du sol, ont par la suite été identifiées. Les premières contrôlent l'intensité et la pénétration continentale de la mousson d'été (Eltahir et Gong, 1996 ; Fontaine et al., 1999), les secondes le cycle de l'eau et la végétation. Ainsi, les anomalies de précipitations d'automne en zone soudano-guinéenne précèdent des anomalies de même signe l'été suivant au Sahel (Philippon et Fontaine, 2002). Par ailleurs, le couplage avec la végétation continentale est nécessaire pour reproduire la variabilité interannuelle et décennale au Sahel dans les modèles (Zeng et al., 1999). Si les travaux d'AMMA ont confirmé ces aspects, ils soulèvent surtout de nouvelles questions. Ainsi, il a été montré que l'indice de végétation « NDVI » du mois de juin est corrélé avec les maximums de NDVI de l'année précédente, laissant supposer un effet mémoire via l'enracinement végétal (Philippon et al., 2007). Mais l'influence de l'eau du sol n'a été retrouvée par expérience avec les modèles de climat que sur quelques semaines au cours de l'automne et pas d'une année sur l'autre comme dans l'observation (Douville et al., 2007). Toutefois, les campagnes de mesures et la modélisation numérique ont bien montré que la variabilité de l'humidité de surface avait un impact sur les flux de surface en augmentant (diminuant) le flux de chaleur latente (sensible) ce qui altère les gradients de température et les vents à méso-échelle (Taylor et al., 2011a).

La phase 2 d'AMMA aura pour objectifs principaux de mieux comprendre les mécanismes de la variabilité décennale en Afrique de 1'Ouest, de séparer cette variabilité du forçage anthropique lié à l'augmentation de la concentration des gaz à effet de serre et de participer aux efforts développés dans le cadre de CMIP5/AR5 du GIEC $^{(1)}$ sur la prévisibilité aux échelles décennale et séculaire. À l'échelle intra-saisonnière, elle étudiera particulièrement l'influence des conditions de surface sur le déclenchement, le cycle de vie et l'efficacité pluviométrique des systèmes convectifs en perspective des missions satellitaires $S M O S^{(2)}$ et Megha-Tropiques.

\section{Modèles couplés et changement climatique en région de mousson africaine}

Actuellement les modèles de climat doivent utiliser de nombreuses simplifications et souffrent de biais importants par rapport à l'observation, particulièrement pour les flux radiatifs, les précipitations et l'eau du sol. Ces incertitudes fortes sont liées en particulier aux choix de paramétrisations des nuages et de la convection, à l'absence de végétation dynamique et au manque de rétroactions liées aux aérosols désertiques (Christensen et al., 2007). La représentation de la mousson africaine dans les modèles couplés est donc partiellement erronée (Cook et Vizy, 2006), avec souvent une pénétration continentale trop faible en été boréal et une variabilité interannuelle peu réaliste en raison des difficultés à simuler les téléconnexions observées avec les grands bassins océaniques et la mer Méditerranée et leur évolution dans le temps. Cela engendre des biais systématiques qui altèrent le réalisme des modes de variabilité dans les modèles de climat. Les travaux d'AMMA ont permis d'apporter quelques précisions en ces matières.

À l'échelle synoptique, par exemple, on a constaté une trop faible activité convective associée à des précipitations pas assez intermittentes, en raison notamment de la difficulté à inhiber la convection par les intrusions d'air sec depuis les couches hautes et moyennes et de la faible résolution spatiale qui lisse la topographie. Aux échelles interannuelles, d'autres problèmes surgissent. Ainsi, dans l'exercice CMIP3/AR4 du GIEC, le lien avec le Pacifique équatorial se produit non en début mais plutôt en fin d'événement de type ENSO (El Niño Southern Oscillation) avec une tendance à la hausse au cours du XXI ${ }^{\mathrm{e}}$ siècle (Joly et Voldoire, 2010). Par ailleurs, quand on regarde les projections sur le $\mathrm{XXI}^{\mathrm{e}}$ siècle, les mécanismes associant la variabilité de la mousson africaine et les températures de surface de mer, et reconnus sur la période récente, ne sont pas stationnaires (Caminade et Terray, 2010), et les modèles produisent des scénarios divergents en termes d'évolution des pluies sur la bande sahélienne.

L'effort d'AMMA a donc été de se focaliser sur cet aspect en questionnant la cohérence des résultats des modèles de climat relatifs à quelques variables et régions clés pour la mousson africaine. Ainsi, tous les modèles dans les scénarios AR4 produisent un réchauffement plus marqué des régions méditerranéennes et sahariennes que des régions Atlantique guinéennes et équatoriales, ce qui favorise le réchauffement accru du continent par rapport aux océans, donc une meilleure circulation de mousson (Paeth et Hense, 2004). Il a été montré qu'à l'horizon 2050, les modèles simulent plutôt des excédents de précipitations sur le Sahel central et oriental associés à un déficit relatif sur les parties ouest, avec des changements spécifiques au sein de la dynamique atmosphérique. Ainsi, en utilisant les 12 modèles couplés de l'AR4 dans le cadre du scénario d'émissions A1B, on a pu documenter les changements de précipitations et de dynamique atmosphérique simulés entre les saisons des pluies 1960-1999 et 2031-2070 : cette information est reproduite sur la figure 3 . Les résultats indiquent plusieurs tendances. D'abord une amélioration significative des saisons des pluies dans le golfe de Guinée (avriljuin) et au Sahel central et oriental (juillet-septembre) : la première est simulée dans 10 modèles sur 12 (figure $3 \mathrm{~g}$ ), la seconde dans 8 modèles sur 12 (figures $3 \mathrm{~h}, 3 \mathrm{i}$ ). À $1^{\prime}$ 'est de $5^{\circ} \mathrm{E}$, les excédents dépassent parfois $30 \%$ et sont associés à une progression vers le nord de la circulation de Hadley-Sud et dans les basses couches de la mousson (figure $3 \mathrm{k}$ ). Les déficits relatifs sur

(1) CMIP : Coupled Models Intercomparison Project. AR : Assesment Report, rapport d'évaluation. GIEC : Groupe intergouvernemental d'experts sur l'évolution du climat.

(2) SMOS : Soil Moisture Ocean Salinity. 
a) Gulf,AMJJ: CTL

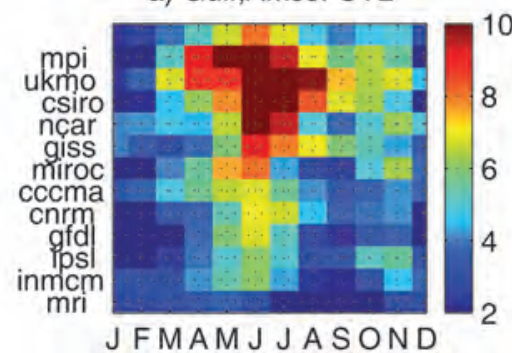

d) Future vs CTL \%

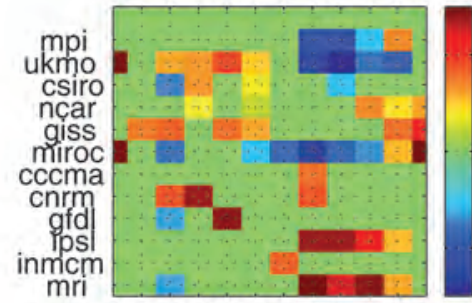

JFMAM J J A SOND

g) Gulf,AMJJ: Future vs CTL \%

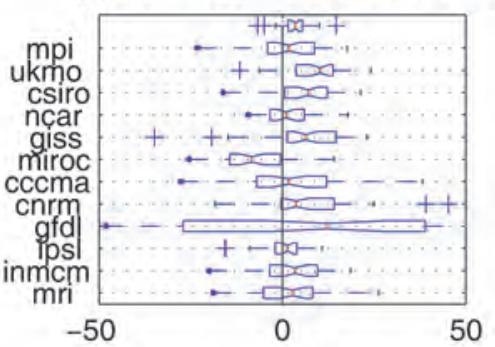

50 b) West, JAS: CTL

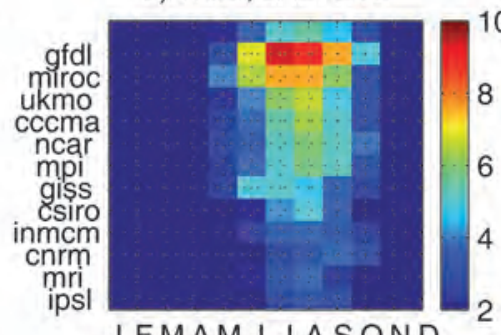

JFMAM J JASOND

e) Future vs CTL \%

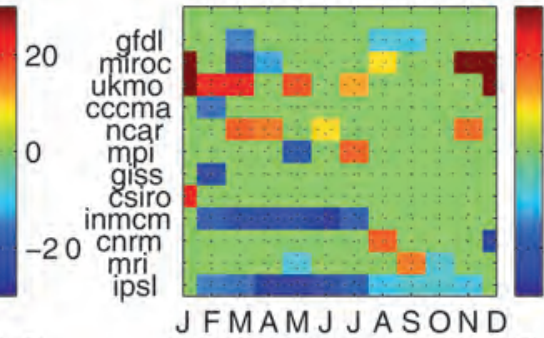

h) West, JAS: Future vs CTL \%

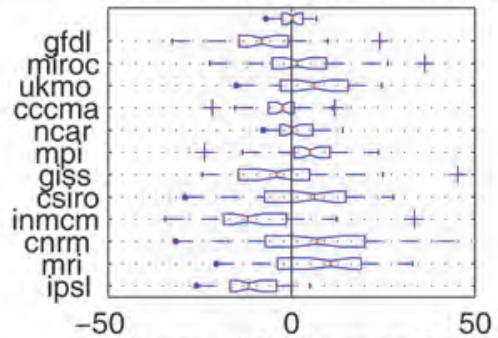

j) West, JAS: Future vs CTL

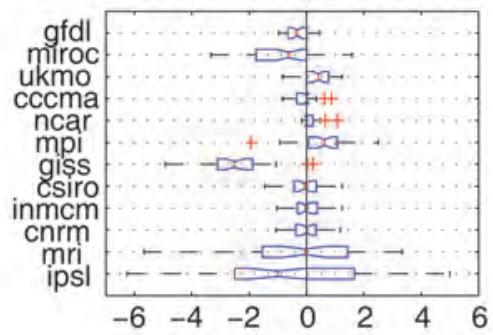

c) East, JAS: CTL

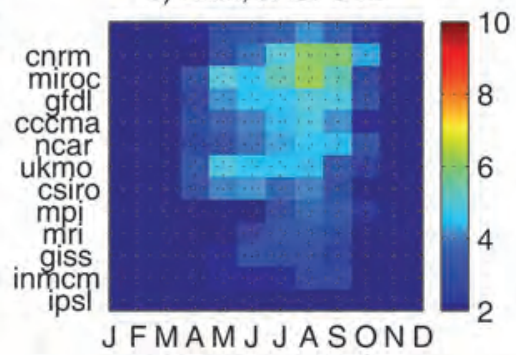

f) Future vs CTL \%

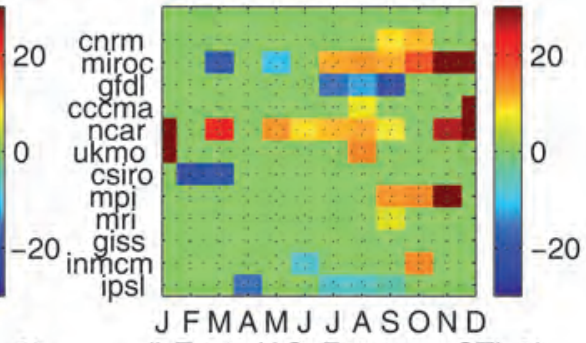

i) East, JAS: Future vs CTL \%

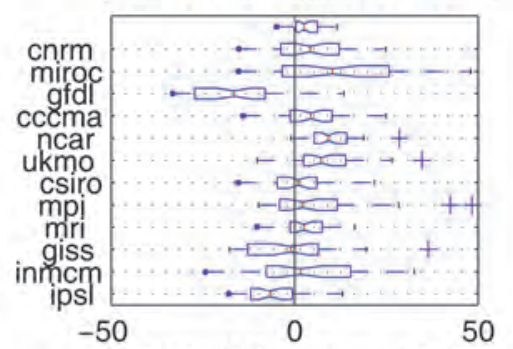

k) East, JAS : Future vs CTL

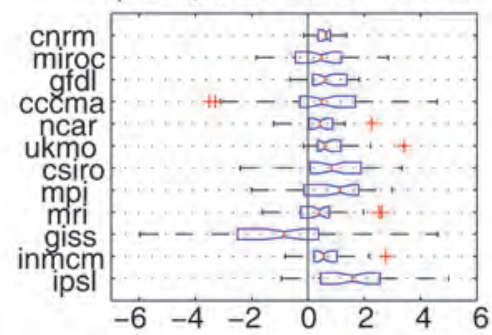

Figure 3 - Simulation d'indices saisonniers en quelques régions-clé (figure issue de Fontaine et al.. 2011).

$(a, b, c)$ Moyennes d'avril à juin (1 1 tre colonne) des précipitations sur le golfe de Guinée $\left[E q-5^{\circ} \mathrm{N} ; 20^{\circ} \mathrm{W}-10^{\circ} \mathrm{E}\right]$, et de juillet à septembre sur les parties ouest $\left[5^{\circ} \mathrm{N}-20^{\circ} \mathrm{N}\right.$; $\left.20^{\circ} \mathrm{W}-0^{\circ}\right]$ et est $\left[5^{\circ} \mathrm{N}-20^{\circ} \mathrm{N} ; 0^{\circ}-30^{\circ} \mathrm{E}\right]$ de l'Afrique de l'Ouest (colonnes 2-3). Les acronymes des modèles (axe Y) sont classés en ordre décroissant par rapport aux cumuls simulés avec la moyenne d'ensemble au sommet.

(d, e, f) Différences significatives en précipitations au seuil $95 \%$ entre les périodes 1960-1999 et 2031-2070 exprimés en pourcentages.

$(\mathrm{g}, \mathrm{h}, \mathrm{i})$ Distributions sur 40 ans de ces différences, avec les quartiles inférieurs et supérieurs ainsi que les médianes, tous exprimés en pourcentages.

(j, k) comme (g, h, i), mais relatif à la position en degrés de latitude du barycentre de la convergence des flux d'humidité à $850 \mathrm{hPa}$ sur l'ouest $\left(15^{\circ} \mathrm{W}-5^{\circ} \mathrm{W} ; 0^{\circ}-14^{\circ} \mathrm{N}\right)$ et le centre-est africains $\left(5^{\circ} \mathrm{W}-20^{\circ} \mathrm{E} ; 0^{\circ}-20^{\circ} \mathrm{N}\right)$. Ici, seuls 11 modèles sur 12 sont considérés, les données d'humidité étant indisponibles dans les sorties UKMO.

l'ouest sont dus à un renforcement du jet d'est africain et à l'existence d'anomalies dans la circulation zonale de type Walker entre l'océan Indien et l'océan Atlantique, favorisant la subsidence de l'air sur la région et l'exportation d'humidité en dehors (Fontaine et al., 2011a ; Monerie et al., 2012).

Les premières analyses des sorties de 8 modèles de 1'exercice CMIP5/AR5 confirment ces aspects. Les excédents sont principalement associés à une augmentation de la convergence du flux d'humidité moyenne sur le Sahel continental, favorisée par le réchauffement sur le continent. La modification des gradients thermiques entraîne une intensification de la cellule de HadleySud et de la convection à l'est du Sahel alors qu'à l'ouest on note une subsidence expliquant les déficits pluviométriques. A moyen terme, Monerie et al. (2012) montrent une covariation nette entre l'augmentation des températures et l'évolution différentielle de la pluviométrie au Sahel. Ces résultats demanderont à être confirmés lorsque l'ensemble des simulations de l'exercice CMIP5 seront disponibles.

\section{L'intérêt de la modélisation régionale du climat}

La modélisation régionale du climat présente l'avantage de simuler la dynamique à des échelles spatiales plus fines que celles des modèles de climat globaux. Elle permet d'étudier plus précisément certains mécanismes associés à la variabilité intra-saisonnière comme celui de l'arrivée de la mousson, et par là même de s'intéresser aux impacts, 
comme par exemple le lien climat/ rendements agricoles dans le cadre du changement climatique. Les modèles régionaux peuvent alors être utilisés comme des outils de désagrégation numérique, suivant certaines précautions cependant.

\section{Pour l'étude de la mousson africaine}

Les évaluations faites de ces modèles ont montré qu'ils sont capables de simuler certains aspects de la mousson avec plus de réalisme que les modèles globaux. Gallée et al. (2004) ont ainsi montré, avec le modèle régional $\mathrm{MAR}^{(1)}$, que la variabilité intrasaisonnière des précipitations pouvait être associée à des phases de renforcement et d'affaiblissement de la circulation de Hadley, eux-mêmes dépendant des gradients d'énergie statique humide. En s'appuyant sur les modèles MAR et MM5 ${ }^{(2)}$, Ramel et al. (2006) et Sijikumar et al. (2006) ont montré que les dates d'installation de la mousson (fin juin en moyenne) étaient très proches de celles observées. Les mécanismes proposés ont mis en avant le rôle essentiel de la dépression thermique saharienne qui se déplace et se renforce rapidement à la fin juin, depuis la région sahélienne en direction du Sahara, via les bilans d'énergie en surface, favorisant ainsi la progression de la mousson. Sultan et Janicot (2003) et Drobinski et al. (2005) avaient auparavant émis l'hypothèse que l'amplification de la dynamique de la dépression saharienne pouvait être due à une interaction avec le relief du Hoggar, ce dernier renforçant la dépression sous le vent du relief. Flaounas et al. (2012) ont tenté de démontrer cette hypothèse en supprimant dans le modèle régional WRF (Weather Research and Forecasting) les reliefs de l'Atlas et du Hoggar. Bien que l'intensité de la dépression soit réduite, en raison d'une diminution du transport d'air chaud

(1) MAR : Modèle atmosphérique régional développé au Laboratoire de glaciologie et géophysique de l'environnement de Grenoble.

(2) MM5 : Mesoscale community model (modèle météorologique non-hydrostatique communautaire développé au National Center for Atmospheric Research).

(3) ENSEMBLES : ENSEMBLE-based Predictions of Climate Changes and their Impacts.

(4) SARRA-H:Système d'analyse régionale de risques agroclimatiques, développé au Centre de coopération internationale en recherche agronomique pour le développement.

(5)ARPĖGE-Climat : Version climatique $d u$ modèle de prévision ARPĖGE de Météo-France. vers le Sahara, impliquant un moindre déplacement vers le nord de la mousson, les simulations n'ont pas montré de modification particulière de la date d'installation de la mousson. Des expériences complémentaires suggèrent que l'arrivée de la mousson pourrait être sous l'influence d'intrusions d'air sec venu de la Méditerranée et affaiblissant momentanément la convection sur l'Afrique de l'Ouest.

\section{Pour les prévisions agronomiques et la détection du changement climatique}

L'agriculture est une activité très dépendante du climat et très concernée par l'amélioration de la prévision climatique pour mieux évaluer et prévoir les rendements. Cependant, les prévisions saisonnières issues de modèles de climat s'accommodent mal des processus d'échelle plus fine (évaporation, transpiration, ruissellement...) directement liés à la production céréalière (Baron et al., 2005). Dans cette optique Oettli et al. (2011) ont examiné les 8 modèles régionaux du projet ENSEMBLES $^{(3)}$ servant à alimenter le modèle agronomique SARRA-H ${ }^{(4)}$. Les résultats montrent que les performances sont très variables suivant les modèles, à cause de déficiences dans la paramétrisation des variables cruciales pour le calcul des rendements. Cette approche multi-modèles permet aussi de quantifier les incertitudes (Hourdin et al., 2010). Cependant, des corrections statistiques de biais sont possibles mais ne permettent pas de restituer la variabilité interannuelle des rendements observés.

Pour mieux estimer les impacts du climat dans le cadre du changement climatique, les modèles régionaux peuvent désagréger les sorties des modèles de climat. Ainsi, Paeth et al. (2009) ont désagrégé des simulations du modèle global ECHAM4 avec le modèle régional REMO, de l'institut Max Planck, jusqu'en 2050. Pour tenir compte des changements d'occupation du sol, ils ont modifié les fractions de végétation et montré que le réchauffement de la surface s'accompagnait d'une réduction de la pluviométrie et d'une augmentation du nombre de séquences sèches. La dynamique atmosphérique d'altitude étant en revanche faiblement modifiée, cela laisse supposer que la dégradation des sols pourrait jouer un rôle plus important que l'augmentation des températures liée à l'augmentation des gaz
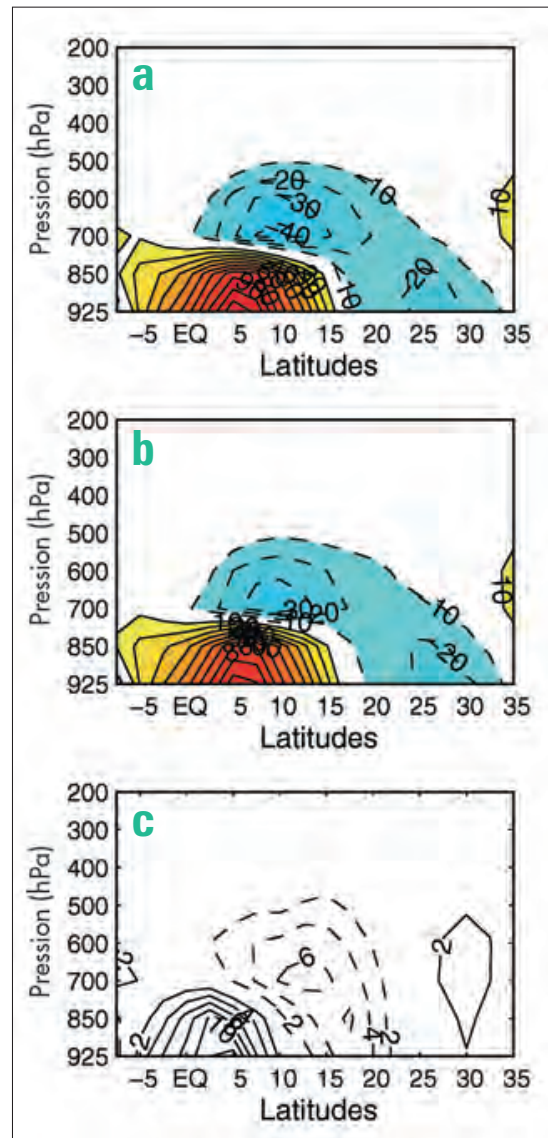

Figure 4 - Coupe verticale du flux d'humidité moyen en juillet-août-septembre entre $10^{\circ} \mathrm{E}$ et $10^{\circ} \mathrm{W}$ pour les simulations WRF forcées par ARPÈGE-Climat entre 2032-2041 (a) et 1981-1990 (b), et leur différence (c). Les flux d'ouest (respectivement est) sont en rouge (resp. bleu). Unités : g. $\mathrm{kg}^{-1} \cdot \mathrm{m} . \mathrm{s}^{-1}$. Figure extraite de Vigaud et al., 2011

à effet de serre, au moins jusqu'en 2050. En revanche, les résultats obtenus par Vigaud et al. (2011) avec le scénario A2, sont assez différents puisqu'ils montrent, après désagrégation des sorties du modèle global ARPĖGEClimat $^{(5)}$ par le modèle régional WRF, une augmentation/diminution de la pluviométrie sur l'est/l'ouest du Sahel à l'horizon 2040. Cette évolution est très proche de celle décrite par Fontaine et al. (2011) et Monerie et al. (2012) à partir de sorties de modèles globaux, mais le modèle régional montre aussi un renforcement du gradient thermique entre régions saharienne et guinéenne particulièrement prononcé, favorisant le flux de mousson (figures $4 \mathrm{a}$ et $4 \mathrm{c}$ ) et une intensification du jet d'est africain (figures $4 \mathrm{~b}$ et $4 \mathrm{c}$ ) qui renforce la divergence d'humidité en moyenne troposphère et contribue à l'augmentation de la convection profonde (Vigaud et al., 2011). Ces mêmes auteurs ont aussi indiqué que les précipitations de fin de saison seraient plus soutenues. Des travaux sur les impacts hydrologiques de ces modifications sont en cours. 
Les résultats obtenus par désagrégation numérique ne sont pas consensuels car ils sont très dépendants des modèles ; c'est pourquoi, dans le cadre du projet CORDEX ${ }^{(1)}$, il est prévu de désagréger plusieurs sorties de modèles globaux avec différents modèles régionaux pour produire des données climatiques à haute résolution permettant de qualifier ces incertitudes.

CORDEX est un exercice coordonné de simulations numériques du climat futur (projections climatiques) mis en œuvre conjointement par plusieurs centres de recherches et de prévisions météorologiques à travers le monde. Ce programme a pour but de produire, avec des modèles climatiques régionaux, des scénarios de changements climatiques aux échelles régionales à des fins d'études d'impacts. Bien que certains de ces modèles présentent un biais fort au large des côtes ouest africaines et sousestiment l'intensité de la pluie sur la bande sahélienne, les premières analyses ont montré qu'ils simulaient bien le gradient méridien de pluviométrie de même que les maxima sur les régions orographiques. Ils représentent également assez bien la migration en latitude de la bande pluvieuse et l'arrivée de la mousson.

\section{Conclusion}

La phase 1 d'AMMA a donné une priorité à l'étude de la variabilité climatique en Afrique de l'Ouest, de l'échelle de l'événement convectif à celle de l'évolution multi-décennale au travers de ses campagnes d'observations intensives et de ses expériences numériques dédiées. Elle s'est attachée notamment à mieux recenser les différents types de forçages intéressant cette gamme large de variabilité. Ainsi, à l'échelle interannuelle et décennale, le rôle des températures de la surface marine et des téléconnexions a été confirmé via la circulation de Walker, associée aux ondes équatoriales d'altitude, et les advections d'humidité depuis notamment la Méditerranée. Toutefois, même à ces échelles longues, la variabilité interne de l'atmosphère apparaît jouer un rôle important. Dans la gamme de variabilité intra-saisonnière, outre les modes caractéristiques de la variabilité à cette échelle, les interactions avec l'albédo de surface, l'eau du sol et la végétation sont déterminantes. Leur effet sur la dynamique atmosphérique est apparu crucial en certaines périodes de l'année : au printemps boréal en influençant les gradients de température et d'énergie statique humide en surface ; en automne en jouant sur l'eau du sol, ce qui peut modifier les états de surface continentaux l'année suivante. L'analyse du changement climatique en Afrique de l'Ouest a été limitée par le fait que les modèles divergent en termes d'évolution des pluies sur la bande sahélienne. Toutefois, il a été montré qu'ils produisent un réchauffement accru du continent par rapport aux océans, favorable à une meilleure circulation de mousson et que, par exemple, à 1'horizon 2050, les modèles de l'AR4 et de l'AR5 simulaient plutôt des excédents de précipitations sur le Sahel central et oriental et un déficit plus à l'ouest par rapport à la normale 1961-1990.
La phase 2 d'AMMA élargit la finalité scientifique en donnant la priorité aux aspects qui ont un fort impact sociétal. Elle s'intéressera ainsi aux mécanismes associés à l'évolution des structures organisées de la convection pour mieux évaluer leur prévisibilité, améliorer les scores de prévision et mettre en place des indicateurs opérationnels, cela en collaboration directe avec les services météorologiques africains afin de mieux diffuser cette information aux utilisateurs et décideurs. De même, les mécanismes propres à la variabilité décennale en Afrique de l'Ouest seront davantage étudiés afin de pouvoir séparer la variabilité naturelle du forçage anthropique impliqué dans l'évolution récente et le changement climatique.

\section{Remerciements}

Les auteurs tiennent à remercier tout particulièrement les contributeurs AMMA, Sophie Bastin, Cyril Caminade, Fabrice Chauvin, Hervé Douville, Cyril Flamant, Emmanouil Flaounas, Nicholas Hall, Frédéric Hourdin, Mathieu Joly, Jean-Philippe Lafore, Christophe Lavaysse, Stephanie Leroux, Paul-Arthur Monerie, Elsa Mohino, Pascal Oettli, Benjamin Sultan, Abdul Khadre Traore, Albin Ullman et Aurore Voldoire

(1) CORDEX : Coordinated Regional climate Downscaling Experiment. 